\title{
Surfactant Incorporated Co Nanoparticles Polymer Composites with Uniform Dispersion and Double Percolation
}

\author{
Tajamal Hussain, ${ }^{1}$ Mirza Nadeem Ahmad, ${ }^{2}$ Aneela Nawaz, ${ }^{1}$ Adnan Mujahid, ${ }^{1}$ \\ Farrukh Bashir, ${ }^{1}$ and Ghulam Mustafa ${ }^{3}$ \\ ${ }^{1}$ Institute of Chemistry, University of the Punjab, New Campus, Lahore 54590, Pakistan \\ ${ }^{2}$ Institute of Chemistry, Government College University, Faisalabad 38030, Pakistan \\ ${ }^{3}$ Suleman Bin Abdullah Aba Al-Khail Centre for Interdisciplinary Research in Basic Sciences, \\ International Islamic University, Islamabad 44000, Pakistan \\ Correspondence should be addressed to Mirza Nadeem Ahmad; pioneeravian@hotmail.com
}

Received 26 September 2017; Revised 31 October 2017; Accepted 7 November 2017; Published 13 December 2017

Academic Editor: Yves Grohens

Copyright (C) 2017 Tajamal Hussain et al. This is an open access article distributed under the Creative Commons Attribution License, which permits unrestricted use, distribution, and reproduction in any medium, provided the original work is properly cited.

\begin{abstract}
Series of Cobalt nanoparticles incorporated polymethylmethacrylate composites in the presence and absence of dodecyl-benzenesulphonic acid (DBSA-CoNPs/PMMA and CoNPs/PMMA, resp.) were synthesized by solution mixing methodology. UV-visible and FTIR techniques were used to confirm the formation of nanocomposite. UV-visible spectra of the composites showed the incorporation of filler particles in the polymer matrix. On the other hand, FTIR spectra indicated the physical interaction between the two phases of the composite. Moreover, the electrical nature of the composites was studied by plotting graphs between electrical conductivity (measured using LCR meter at $100 \mathrm{kHz}$ ) and contents of the filler particles as introduced in the polymer matrix. An increase in electrical conductivity was first observed with increasing filler concentration up to the critical percolation threshold value $(0.5 \%$ for DBSA-CoNPs/PMMA and $1 \%$ for CoNPs/PMMA), which then dropped upon further increments in the filler content. However, at higher concentrations, a second jump in the conductivity was observed in case of DBSA-CoNPs/PMMA composites.
\end{abstract}

\section{Introduction}

Nowadays, extensive research work has been conducted in the field of nanotechnology and nanoscience because of their versatile applications. [1]. Nanoscience is an emerging field and its impact is expected to be spread all over the world. An important aspect is that its sphere is not limited to the materials and its application but expands to life sciences as well [2]. Keeping in view high flexibility in polymers and ease in their processing, other properties of polymers were targeted to boost by doping of metal nanoparticles (NPs) such as cobalt, nickel, silver, and gold NPs [3, 4]. Some polymers are semiconductors such as polyaniline (PANI) and some are nonconductors like poly-methyl methacrylate (PMMA). After the discovery of conductive polymers like poly-acetylene conductor because of conjugated $\pi$ bonds, these polymers are major key point for development of nanotechnology in various fields of science $[5,6]$. Composite nanomaterials exhibit certain collegial properties between polymer and NPs, making them dormant candidates for application in diverse fields serving in catalysis, sensors, displays, data storage materials, polymer films, and so forth [7, 8]. A major issue related to composite formation is that problems occur during the incorporation of filler in the matrix. Common concern in this regard is nonhomogenous dispersion of the particles or agglomerate formation of the filler entities. By doping of some additives or modification of the filler to develop the interaction with matrix, aggregation of nanoparticles can be prevented, and it is very necessary for its homogenous dispersion [9-11]. Homogeneous dispersion of the filler in the polymer matrix is an essential requirement to impart the electrical and mechanical features of the fillers to 
the polymer composite [12]. Low value of electrical percolation is the index of good dispersion [13]. It is also observed that shape of the percolation curve and value of percolation threshold depend strongly upon different factors like temperature, pressure, aspect ratio of fillers, additives used, and method of preparation [11, 14-16].

In the present work dodecyl-benzene-sulphonic acid (DBSA) was used for the synthesis of PMMA based composites along the CoNPs to attain their homogeneous and uniform dispersion to get the maximum out of it. For this study PMMA was selected as matrix because of its unique characteristics $[5,17]$. It is low-cost polymer with extraordinary environmental stability and ability to form thin layers with good mechanical strength. It is believed that DBSA as surfactant will play its role in the better dispersion of CoNPs in PMMA so that characteristics of the CoNPs can be imparted in PMMA more effectively [18-20]. This will help in reducing the chances of agglomerate formation of CoNPs. Synthesized composites have been characterized by UV-visible and FTIR spectroscopy. Electrical character of the PMMA based CoNPs composites prepared with DBSA (DBSA-CoNPs/PMMA) and without the incorporation of DBSA (CoNPs/PMMA) was studied by using conductivity data measured by LCR meter.

\section{Experimental Work}

2.1. Reagents and Instruments. All the chemicals were used as purchased without further processing and purification except solvent. UV-Visible double beam spectrophotometer (PG Instruments Model: UVD-T90+) and Fourier Transform Infrared (FTIR) Spectroscopy (Agilent FT-IR model: CARY630: range $4000-650 \mathrm{~cm}^{-1}$ ) were used to characterize the synthesis of the composites. LCR meter (Precision LCR meter E4980AL, $20 \mathrm{~Hz}-1 \mathrm{MHz}$ ) was used to measure the conductance of the composites.

2.2. Synthesis of CoNPs. A dark blue solution was formed after dissolving cobalt salt, $\mathrm{CoCl}_{2} \cdot 6 \mathrm{H}_{2} \mathrm{O}$, in ethanol and then the mixture of hydrazine hydrate $\left(\mathrm{N}_{2} \mathrm{H}_{4} \cdot \mathrm{H}_{2} \mathrm{O}\right)$ and sodium hydroxide $(\mathrm{NaOH})$ was put in this dark blue solution at room temperature. After about one minute, gray solid particles appear as a result of quick reduction of Co ions. The suspended gray particles can be precipitated by placing a magnet under the container. After completely washing with distilled water, ethanol, absolute ethanol to remove hydrazine, sodium, and chlorine ion, the sample was kept in absolute ethanol to avoid oxidation.

2.3. Synthesis of CoNPs/PMMA Composite. For the synthesis of CoNPs/PMMA composites, $0.025 \mathrm{~g}$ CoNPs were dispersed in $50 \mathrm{~mL}$ of chloroform containing $1.76 \mathrm{~g}$ of PMMA followed by stirring of 40 minutes. This mixture was ultrasonicated for 1 hour to obtain a uniform dispersion of CoNPs. Thin polymer film was casted by pouring the solution into a Petridish and allowing the chloroform to evaporate over several hours. The same experiment was performed with varying concentrations of CoNPs and PMMA to obtain composites with different compositions.
2.4. Synthesis of DBSA-CoNPs/PMMA Composite. In this method, DBSA was used additionally as surfactant. $1 \mathrm{~g}$ of DBSA was stirred in $200 \mathrm{~mL}$ of distilled water for 15 minutes. Then $1 \mathrm{~g}$ of CoNPs was added in the above solution and stirred for 50 minutes. The product was subjected to ultrasonic treatment for 50 minutes. The suspension was covered with perforated aluminium foil and kept for 14 hours. The resultant product was dried at $60^{\circ} \mathrm{C}$ for 14 hours after filtration and wash.

Afterwards, $0.05 \mathrm{~g}$ of the above product was dispersed in $50 \mathrm{~mL}$ chloroform containing $1.76 \mathrm{~g}$ of PMMA along constant stirring of 40 minutes. Then this mixture was ultrasonicated for one hour to obtain a uniform dispersion of DBSA-CoNPs. Thin films were formed by pouring the solutions into a Petridish and allowing the chloroform to evaporate for several hours. The same experiment was performed with varying concentrations of DBSA-CoNPs nanoparticles and PMMA to obtain composites with different compositions. Whole experimental scheme adopted in this research work is best explained by graphical presentation given in Figure 1 .

\section{Results and Discussion}

3.1. UV-Vis Spectroscopy. UV-Vis spectra of pure PMMA, CoNPs/PMMA, and DBSA-CoNPs/PMMA composites are given in Figure 2. The spectrum of pure PMMA shows maximum absorbance around $260 \mathrm{~nm}$, which is ascribed to characteristics absorption curve of PMMA. With addition of CoNPs with and without DBSA, in the PMMA matrix, the absorption is increased showing incorporation of CoNPs in the structure of PMMA. This increase in the absorption intensity was more pronounced in the case when DBSA was added as an additive which clearly indicated that DBSA helped in better incorporation of CoNPs $[4,19,21]$. It can be noted that shift in the position of maximum absorption is not significant.

3.2. FTIR Spectroscopy. FTIR spectra of pure PMMA, CoNPs/PMMA, and DBSA-CoNPs/PMMA composites are shown in Figure 3. The FTIR spectrum of PMMA shows characteristic peaks of various functional groups present in it. Peak at $2950 \mathrm{~cm}^{-1}$ corresponds to C-H stretching vibrations and a peak corresponding to $\mathrm{C}=\mathrm{O}$ appears at $1721 \mathrm{~cm}^{-1}$. Strong bands present at 1449 and $1437 \mathrm{~cm}^{-1}$ originate from the $\mathrm{O}-\mathrm{CH}_{3}$ bending vibrations. Peak at $1146 \mathrm{~cm}^{-1}$ corresponds to the $\mathrm{C}-\mathrm{O}$ in PMMA structure while bending vibrations of $\mathrm{C}-\mathrm{H}$ exhibited absorption at 843,914 , and 986 $\mathrm{cm}^{-1}$ and peak at $749 \mathrm{~cm}^{-1}$ appeared due to polymer chains vibrations. On comparing FTIR spectrum of PMMA with those of its composites no significant difference in peak patterns was observed. This indicates no chemical reaction of filler and DBSA with matrix took place. All the chemical functional groups were intact since incorporation of the filler in matrix is physical.

3.3. Electrical Character. To investigate the electrical character of the series of the DBSA-CoNPs/PMMA and CoNPs/ PMMA composites, their conductance has been measured using LCR meter. The trend of electrical conductivity as a 


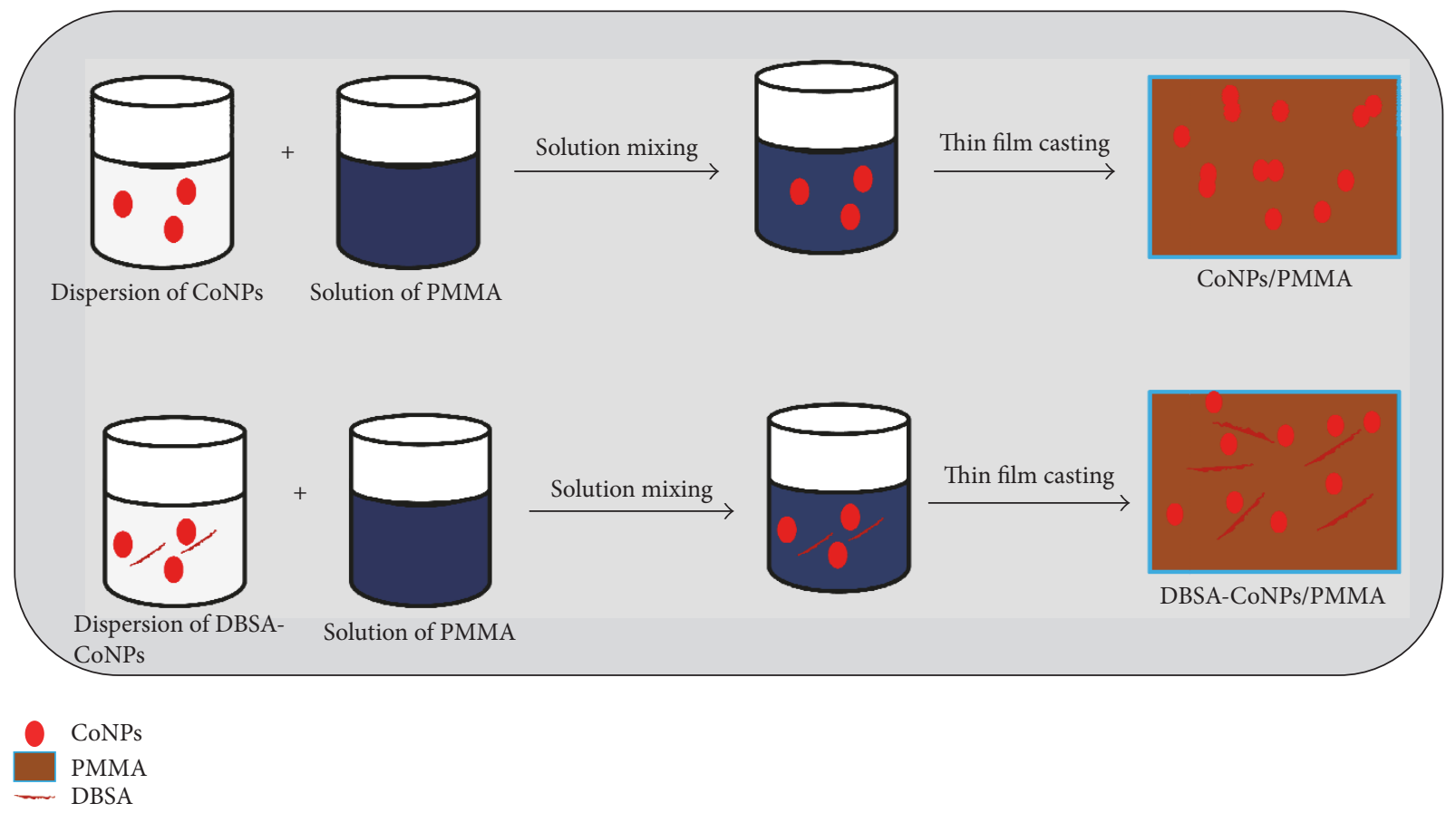

FIGURE 1: Graphical presentation of experimental scheme.

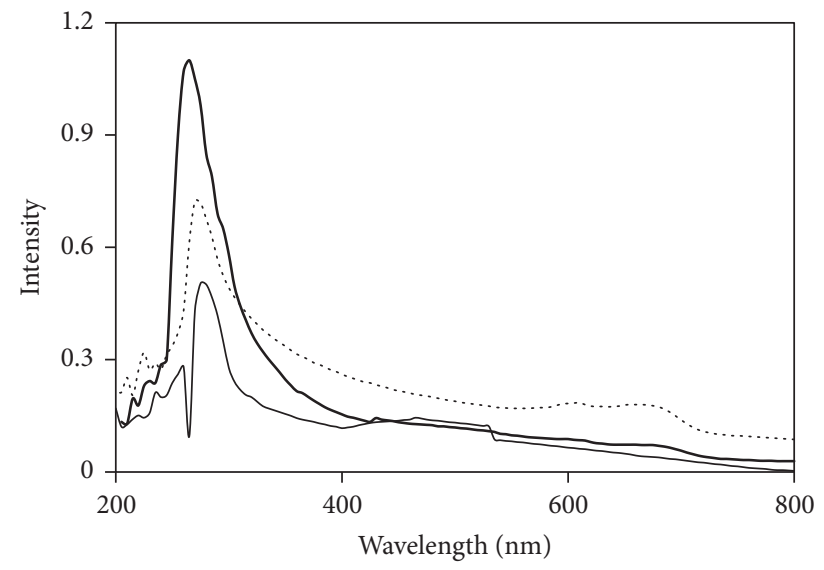

Figure 2: UV-visible spectra of pure PMMA (thin line), CoNPs/ PMMA composites (dotted line), and DBSA-CoNPs/PMMA composites (thick line).

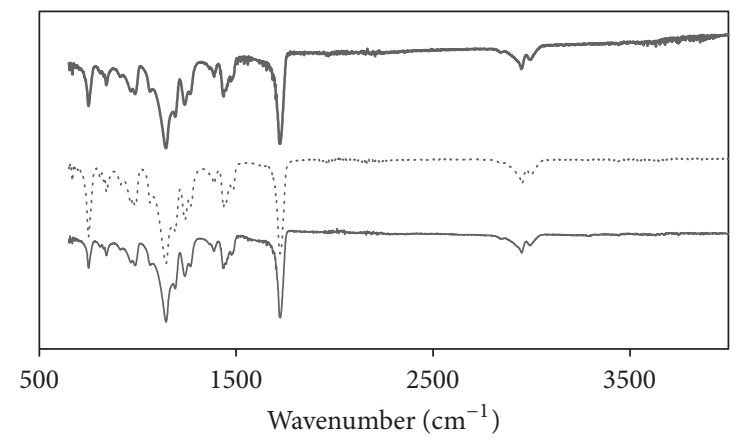

Figure 3: FTIR spectra of pure PMMA (thin line), CoNPs/PMMA composites (dotted line), and DBSA-CoNPs/PMMA composites (thick line).

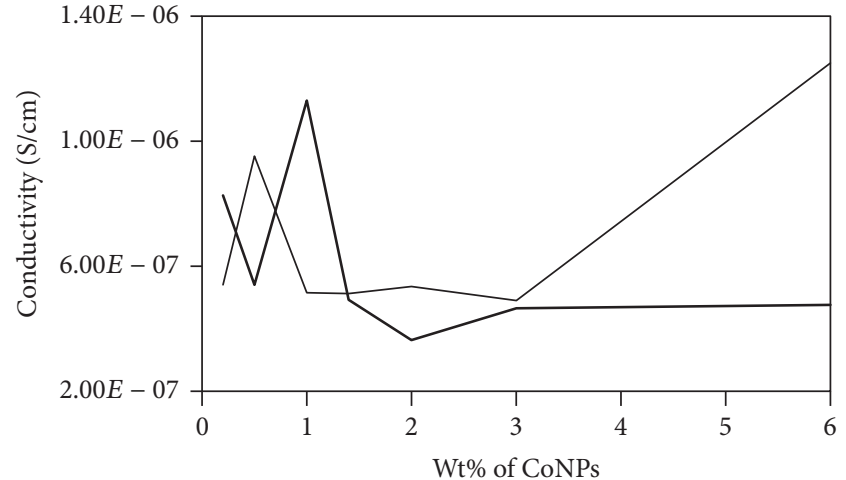

FIGURE 4: Plot of conductance of DBSA-CoNPs/PMMA (thin line) and CoNPs/PMMA (thick line) as a function of contents of CoNPs.

function of filler contents has been portrayed in Figure 4. Critical percolation threshold is minimum concentration at which a network of fillers is just developed which is responsible for the flow of current. Upon formation of the network, current, which is stored due to micro size capacitors consisting of conductor, filler, and insulator matrix, flows away. This results in sudden rise in the value of the observed conductivity $[22,23]$. This high level of observed conductivity lasts until network exists. It is often observed that, soon after attaining the maximum value of the current, further increase in the contents of fillers causes reagglomeration of the fillers which results in a decreased value of conductivity. Percolation phenomena occur in both types of the composites and can be seen in Figure 4. Value of the critical threshold for percolation can be determined with the help of (1) and (2) by using data of conductivity and dielectric constant, respectively, as 


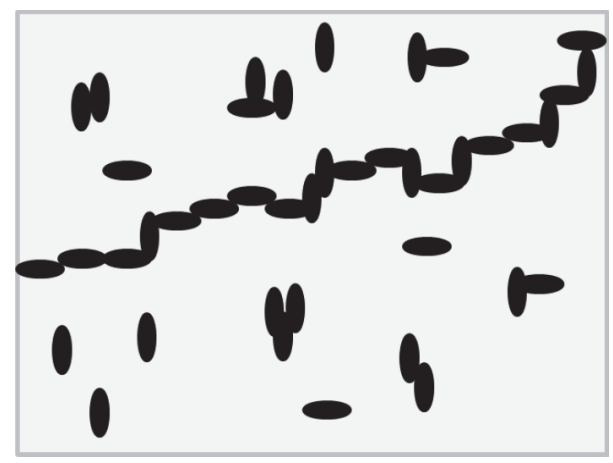

CoNPs

PMMA

(a)

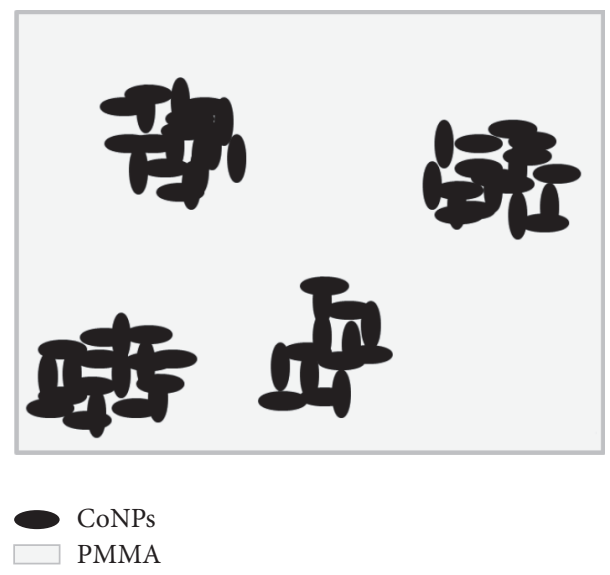

(c)

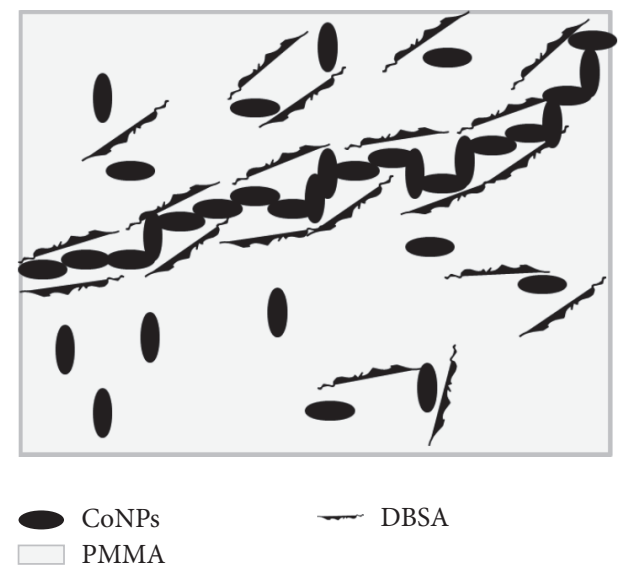

(b)

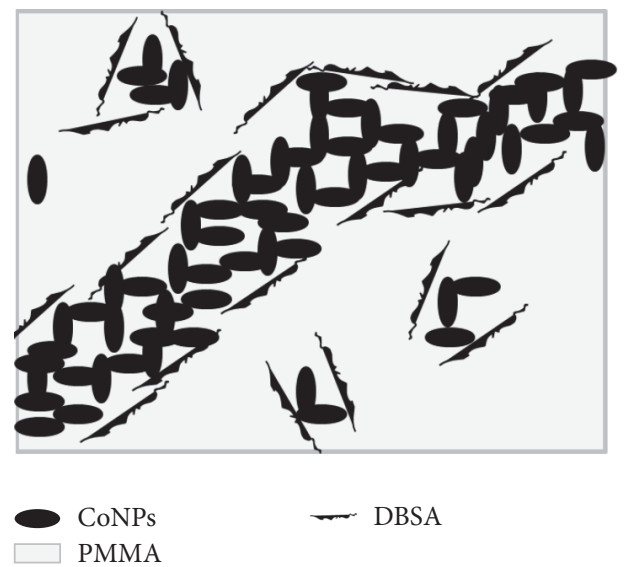

(d)

FIGURE 5: Graphical presentation of pattern of dispersion of fillers at percolation for CoNPs/PMMA (a) and DBSA-CoNPs/PMMA (b) and at filler contents higher than percolation threshold for CoNPS/PMMA (c) and DBSA-CoNPs/PMMA (d).

a function of contents of filler [14]. Percolation behavior is quite different for both types of the composites. Value of the percolation threshold, calculated using data of conductivity, is lower for DBSA-CoNPs/PMMA composites as compared to CoNPs/PMMA composites, while value of the maximum conductivity is higher for CoNPs/PMMA composites than for DBSA-CoNPs/PMMA. Value of critical percolation threshold by using (1) is around 0.5 weight percent for DBSACoNPs/PMMA while that for CoNPs/PMMA is around 1 weight percentage of CoNPs. Values of percolation threshold for both the systems improved from previously reported percolation values for polymer composite incorporated conducting materials as filler [24]. On the parallel side a relatively high maximum value in the conductivity was observed for CoNPs/PMMA composites. Low value of percolation for DBSA-CoNPs/PMMA as compared to CoNPs/PMMA indicated that DBSA helped in network formation by reducing the chances of reagglomeration of the filler particles being a surfactant. The value of maximum conductivity was also reduced, due to the nonconducting nature of the surfactant which interrupted the electrical conductivity. This phenomenon of percolation can be well understood with the aid of Figures 5(a) and 5(b).

Upon further increase in the filler contents beyond the percolation threshold values, decrease in the conductivity was observed which is due to breaking of conductive network and reagglomeration of filler particles for both types of composites. At higher region of the filler contents, another jump of conductivity was seen only for DBSA-CoNPs/PMMA composites, shown in Figure 4. This second jump of conductivity may have occurred due to the aid provided by DBSA to avoid the formation of large agglomerated bundles and alignment of smaller agglomerated bundles of filler particles in such a way that they form a conductive network. Now, the maximum conductivity of the DBSA-CoNPs/PMMA composite is higher as compared to that of the same composite when it has relatively lower concentration of filler. Since the conductive path of fillers now formed consists of relatively larger bundles of fillers as compared to the conductive path formed in the same composite previously at lower filler concentration, maximum conductivity of the composite is comparatively higher. However, in case of CoNPs/PMMA composite, no 


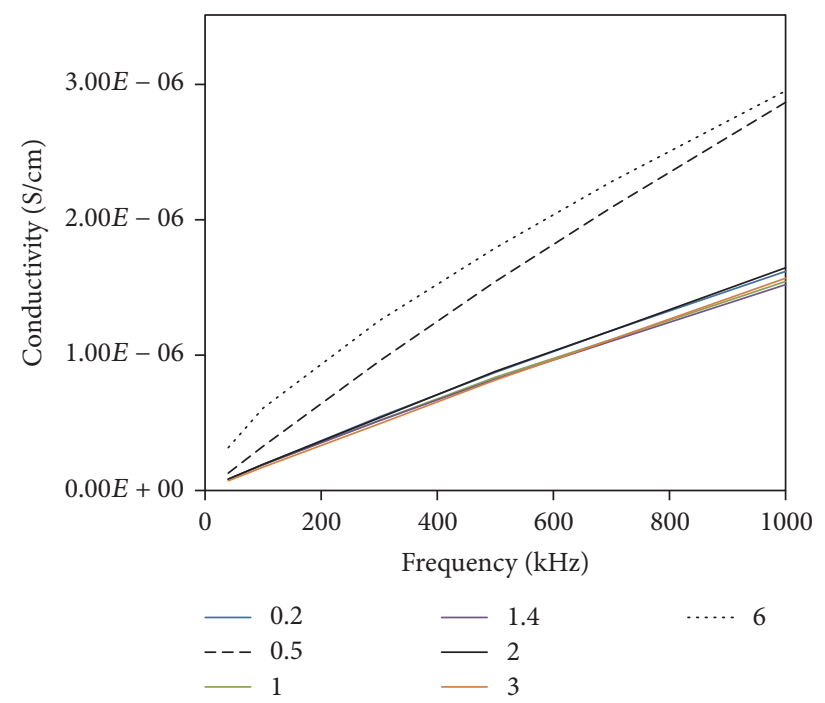

(a)

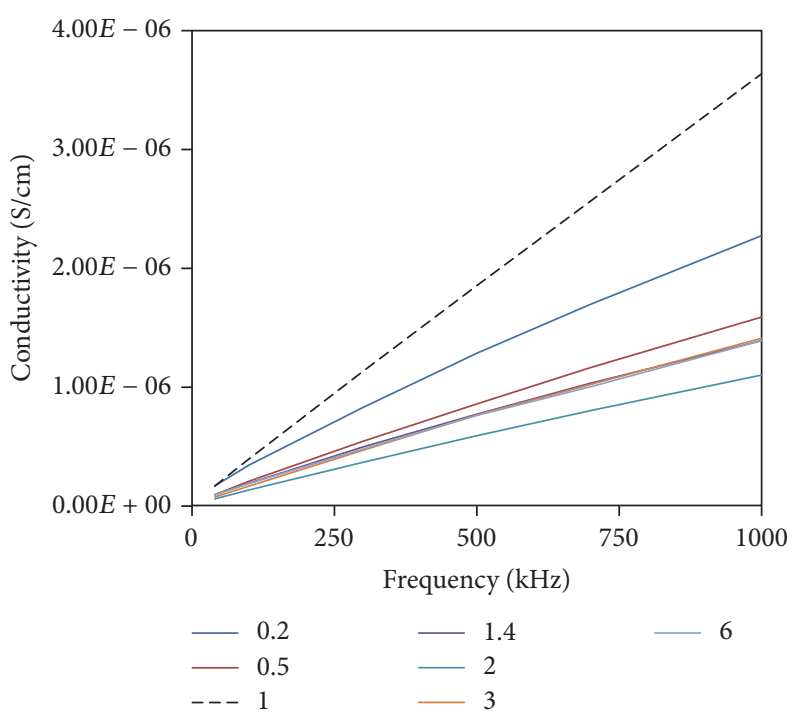

(b)

FIGURE 6: Frequency dependence electrical conductivity for (a) DBSA-CoNPs/PMMA and (b) CoNPs/PMMA composites for different weight percentage of CoNPs.

increase in conductivity at higher filler concentration is observed due to the absence of DBSA that resulted in the formation of large agglomerated parts of filler in the polymer matrix. So this can be concluded from whole discussion that presence of a surfactant in polymer nanocomposites may lead to an enhanced electrical conductivity at higher filler concentrations. Such kind of pattern of dispersion for high contents of filler for both types of composites can be best illustrated with help of Figures 5(c) and 5(d):

$$
\begin{aligned}
& \sigma \propto\left(f_{\text {filler }}-f_{c}\right)^{t}, \quad \text { for } f_{c} \leq f_{\text {filler }}, \\
& \varepsilon \propto\left(f_{\text {filler }}-f_{c}\right)^{-q}, \quad \text { for } f_{c} \geq f_{\text {filler }} .
\end{aligned}
$$

Interesting frequency to conductivity relationship was found for different weight percentages of CoNPs in the presence and absence of DBSA and these results of frequency dependence conductivity for different weight percentages are given in Figure 6. Frequency dependent conductivity relationship is often analyzed in terms of resistance-capacitance percolation model $[25,26]$. According to that model, it is assumed that AC conductivity consists of two contributions. One is frequency independent which is named as DC or ohmic conductivity. The other part which is frequency dependent comes from dielectric dispersion. It can be seen from Figure 6 that contribution of second type of conductivity was more pronounced as compared to first type since conductivity is very much dependent upon frequency. This character is more dominant for composites with filler fraction around percolation threshold; curves are highlighted semisolid lines in Figure 6. This relationship of frequency dependent conductivity for both types of composites is qualitatively similar. It was reported elsewhere that effect of frequency can be classified into three categories on broader scale for polymer composites with CNTs as filler [27]: first, composites with concentrations of filler lower than percolation; second, near to percolation; and third, higher than percolation. It is really very complex relation to analyze. For all three regions, dependence of conductivity as function of frequency was different. Moreover, quantitatively, this dependence of electrical conductivity on frequency was not uniform for whole range of frequency. But in present case such an effect was not found. Curve belongs to each composite with different contents of filler as shown in Figure 6 and has almost constant slope for whole range of frequency.

\section{Conclusion}

Series of PMMA based CoNPs polymer composites with DBSA and without DBSA have been prepared. Agglomeration of the filler in the polymer matrix is a big dilemma and shows encumbrance to attain the homogeneous and uniform dispersion of the filler particles. Addition of the DBSA additive, along the CoNPs, played an important role in uniform dispersion of the fillers in the matrix and achieving low percolation value as compared to that of series of composites where no such additive was added. DBSA also helped in establishing second conductivity network built of agglomerated CoNPs. That network was responsible for second jump in conductivity which is relatively high as compared to first jump.

\section{Conflicts of Interest}

The authors declare no conflicts of interest regarding the research project.

\section{Authors' Contributions}

Tajamal Hussain and Aneela Nawaz contributed equally to this work. 


\section{Acknowledgments}

University of the Punjab, Lahore, is highly acknowledged for research grant to carry out this work.

\section{References}

[1] J. S. Hrkach, M. T. Peracchia, A. Domb, N. Lotan, and R. Langer, "Nanotechnology for biomaterials engineering: structural characterization of amphiphilic polymeric nanoparticles by $1 \mathrm{H}$ NMR spectroscopy," Biomaterials, vol. 18, no. 1, pp. 27-30, 1997.

[2] J. S. A. Bhat, "Concerns of new technology based industriesthe case of nanotechnology," Technovation, vol. 25, no. 5, pp. 457-462, 2005.

[3] A. Hashim, M. K. Al-Khaykanee, and A. Mohammad, "Characterization of (PMMA-CoCl 2) composites," Journal of Babylon University, vol. 21, 2013.

[4] E. Tang, G. Cheng, X. Pang, X. Ma, and F. Xing, "Synthesis of nano- $\mathrm{ZnO} /$ poly(methyl methacrylate) composite microsphere through emulsion polymerization and its UV-shielding property," Colloid and Polymer Science, vol. 284, no. 4, pp. 422-428, 2006.

[5] M. Aghazadeh and F. Aghazadeh, "Electrical conductivity property study of polyanilinecobalt nanocomposite," Journal of Applied Chemical Research, vol. 7, no. 3, pp. 47-55, 2013.

[6] T. K. Sarma and A. Chattopadhyay, "One pot synthesis of nanoparticles of aqueous colloidal polyaniline and its $\mathrm{Au}$ nanoparticle composite from monomer vapor," The Journal of Physical Chemistry A, vol. 108, no. 39, pp. 7837-7842, 2004.

[7] A. Liu, L. H. Bac, J.-S. Kim, B.-K. Kim, and J.-C. Kim, "Synthesis and characterization of conducting polyaniline-copper composites," Journal of Nanoscience and Nanotechnology, vol. 13, no. 11, pp. 7728-7733, 2013.

[8] T. Hussain, S. Jabeen, K. Shehzad et al., "Polyaniline/silver decorated-MWCNT composites with enhanced electrical and thermal properties," Polymer Composites, 2016.

[9] A. Gedanken, "Doping nanoparticles into polymers and ceramics using ultrasound radiation," Ultrasonics Sonochemistry, vol. 14, no. 4, pp. 418-430, 2007.

[10] M. S. P. Shaffer and A. H. Windle, "Fabrication and characterization of carbon nanotube/poly(vinyl alcohol) composites," Advanced Materials, vol. 11, no. 11, pp. 937-941, 1999.

[11] F. Bashir, T. Hussain, A. Mujahid et al., "Tailoring electrical and thermal properties of polymethyl methacrylate-carbon nanotubes composites through polyaniline and dodecyl benzene sulphonic acid impregnation," Polymer Composites, 2017.

[12] S. M. Reda and S. M. Al-Ghannam, "Synthesis and electrical properties of polyaniline composite with silver nanoparticles," Advances in Materials Physics and Chemistry, vol. 2, pp. 75-81, 2012.

[13] R. Ramasubramaniam, J. Chen, and H. Liu, "Homogeneous carbon nanotube/polymer composites for electrical applications," Applied Physics Letters, vol. 83, no. 14, pp. 2928-2930, 2003.

[14] Z. Dang, K. Shehzad, J. Zha, T. Hussain, N. Jun, and J. Bai, "On refining the relationship between aspect ratio and percolation threshold of practical carbon nanotubes/polymer nanocomposites," Japanese Journal of Applied Physics, vol. 50, no. 8R, p. 080214, 2011.

[15] K. Shehzad, A. Ul-Haq, S. Ahmad et al., "All-organic PANIDBSA/PVDF dielectric composites with unique electrical properties," Journal of Materials Science, vol. 48, no. 10, pp. 37373744, 2013.
[16] J. K. W. Sandler, J. E. Kirk, I. A. Kinloch, M. S. P. Shaffer, and A. H. Windle, "Ultra-low electrical percolation threshold in carbon-nanotube-epoxy composites," Polymer Journal, vol. 44, no. 19, pp. 5893-5899, 2003.

[17] S. Devikala and P. Kamaraj, "Development of polymethylmethacrylate based composite for gas sensing application," $E$ Journal of Chemistry, vol. 8, no. 1, pp. S165-S170, 2011.

[18] P. Linganathan and J. M. Samuel, "Synthesis, characterization and electrical conductivity of poly (2-Chloroaniline)/MMT and poly (2-Chloroaniline)/Na-Bentonite Nano composites in the presence of surfactants," International Journal Of Scientific \& Technology Research, vol. 3, 2014.

[19] J. Vilčáková, R. Moučka, P. Svoboda et al., "Effect of Surfactants and manufacturing methods on the electrical and thermal conductivity of carbon nanotube/silicone composites," Molecules, vol. 17, no. 11, pp. 13157-13174, 2012.

[20] S.-J. Su and N. Kuramoto, "Synthesis of processable polyaniline complexed with anionic surfactant and its conducting blends in aqueous and organic system," Synthetic Metals, vol. 108, no. 2, pp. 121-126, 2000.

[21] M. A. Reyes-Acosta, A. M. Torres-Huerta, M. A. DomínguezCrespo, A. I. Flores-Vela, H. J. Dorantes-Rosales, and J. A. Andraca-Adame, "Thermal, mechanical and UV-shielding properties of poly(methyl methacrylate)/cerium dioxide hybrid systems obtained by melt compounding," Polymer, vol. 7, no. 9, pp. 1638-1659, 2015.

[22] L. Terlemezyan, M. Mihailov, and B. Ivanova, "Electrically conductive polymer blends comprising polyaniline," Polymer Bulletin, vol. 29, no. 3-4, pp. 283-287, 1992.

[23] M. Sumita, K. Sakata, S. Asai, K. Miyasaka, and H. Nakagawa, "Dispersion of fillers and the electrical conductivity of polymer blends filled with carbon black," Polymer Bulletin, vol. 25, no. 2, pp. 265-271, 1991.

[24] C. McClory, T. McNally, M. Baxendale, P. Pötschke, W. Blau, and M. Ruether, "Electrical and rheological percolation of PMMA/MWCNT nanocomposites as a function of CNT geometry and functionality," European Polymer Journal, vol. 46, no. 5, pp. 854-868, 2010.

[25] A. R. von Hippel and S. O. Morgan, "Dielectrics and waves," Journal of The Electrochemical Society, vol. 102, no. 3, p. 68C, 1955.

[26] A. K. Jonscher, "Dielectric relaxation in solids," Journal of Physics D: Applied Physics, vol. 32, no. 14, pp. R57-R70, 1999.

[27] K. Shehzad, Z.-M. Dang, M. N. Ahmad et al., "Effects of carbon nanotubes aspect ratio on the qualitative and quantitative aspects of frequency response of electrical conductivity and dielectric permittivity in the carbon nanotube/polymer composites," Carbon, vol. 54, pp. 105-112, 2013. 

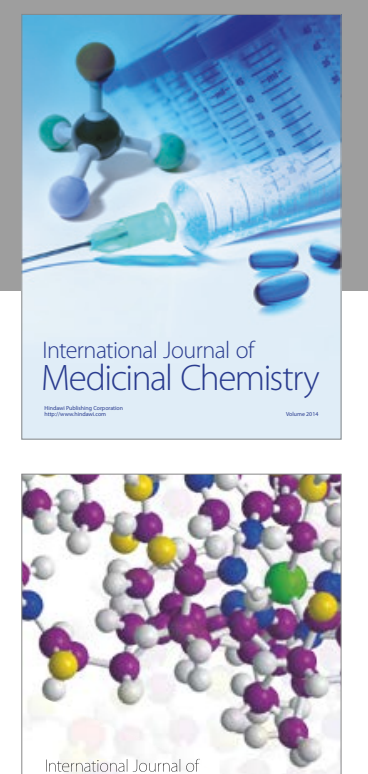

Carbohydrate Chemistry

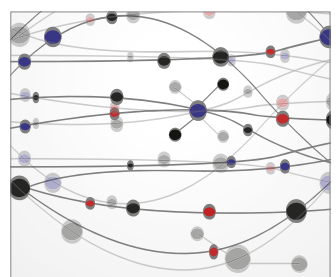

The Scientific World Journal
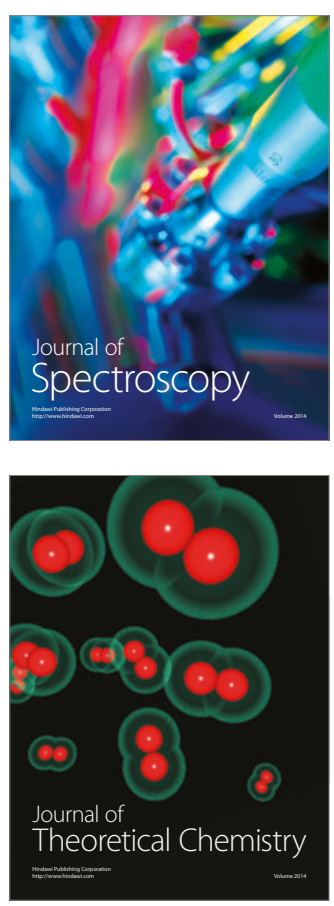
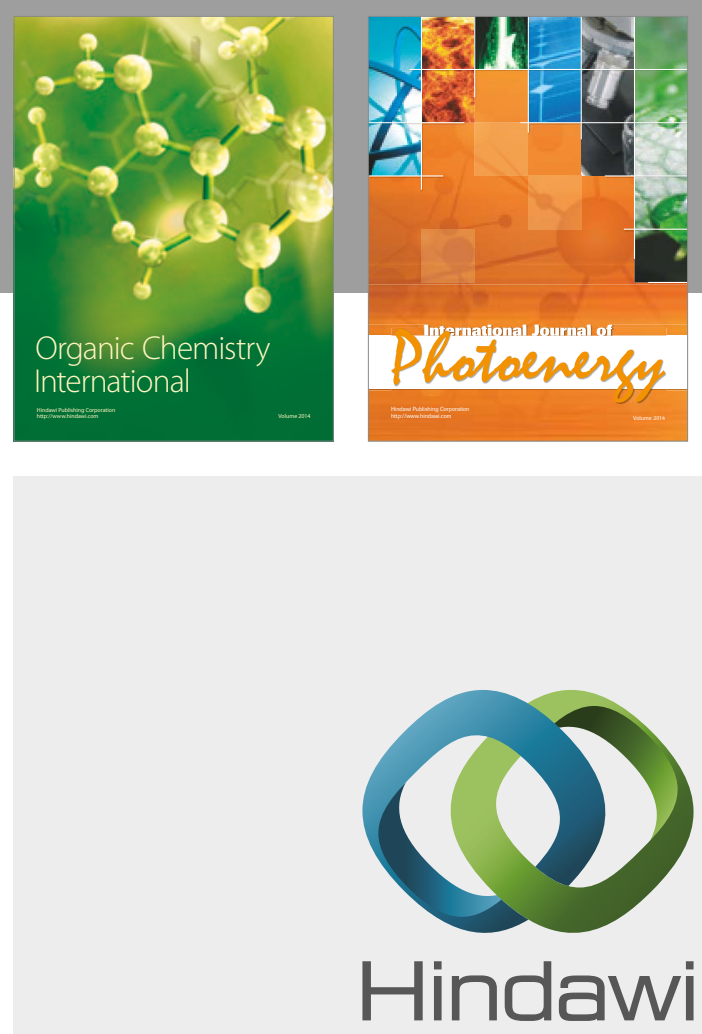

Submit your manuscripts at

https://www.hindawi.com

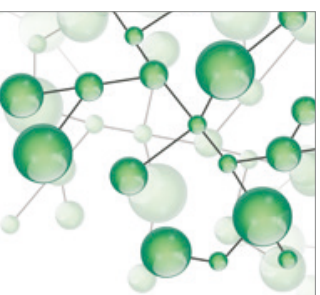

International Journal of

Inorganic Chemistry

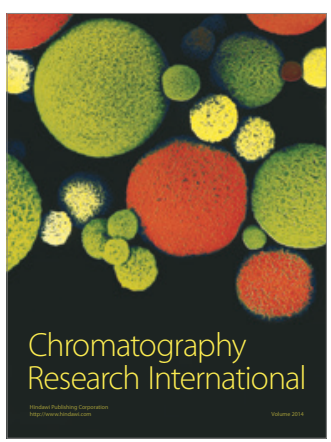

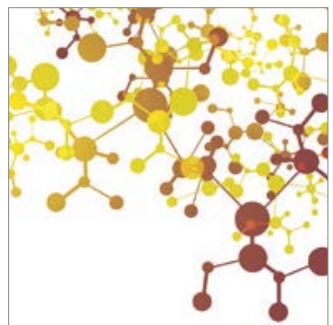

Applied Chemistry
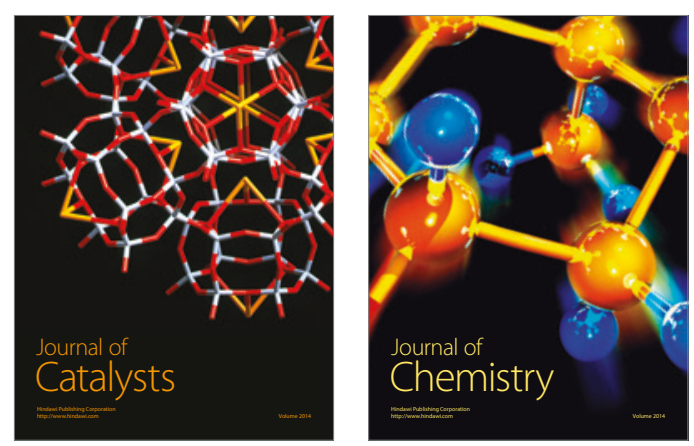
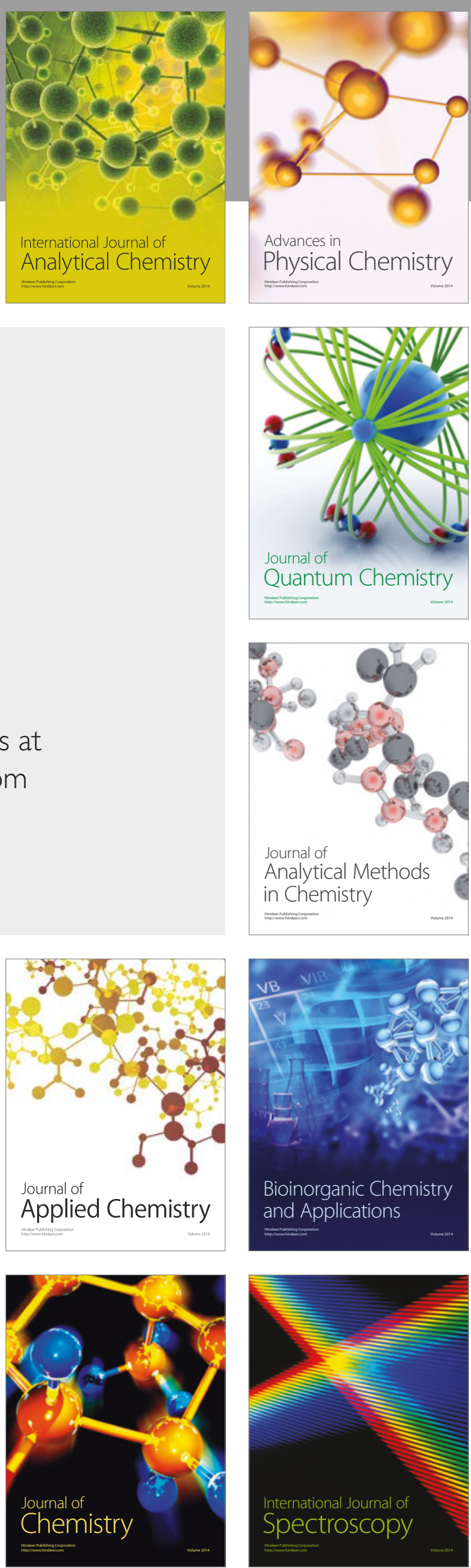